\title{
Médiévales
}

Langues, Textes, Histoire

57 | automne 2009

Langages politiques, $\mathrm{XII}{ }^{\mathrm{e}}-\mathrm{XV}^{\mathrm{e}}$ siècle

\section{L'audience des prophéties de Merlin: entre rumeurs populaires et textes savants}

The Audience of the Prophecies of Merlin: from Popular Rumour to Scholarly Texts

\section{Catherine Daniel}

\section{(2) OpenEdition}

Journals

Édition électronique

URL : https://journals.openedition.org/medievales/5800

DOI : 10.4000/medievales. 5800

ISSN : $1777-5892$

Éditeur

Presses universitaires de Vincennes

Édition imprimée

Date de publication : 20 décembre 2009

Pagination : $33-51$

ISBN : 978-2-84292-241-2

ISSN : 0751-2708

\section{Référence électronique}

Catherine Daniel, «L'audience des prophéties de Merlin: entre rumeurs populaires et textes savants »,

Médiévales [En ligne], 57 | automne 2009, mis en ligne le 18 janvier 2012, consulté le 22 avril 2022.

URL : http://journals.openedition.org/medievales/5800; DOI : https://doi.org/10.4000/medievales.

5800 
Médiévales 57, automne 2009, p. 33-52

Catherine DANIEL

\section{L'AUDIENCE DES PROPHÉTIES DE MERLIN : ENTRE RUMEURS POPULAIRES ET TEXTES SAVANTS}

L'étude des prophéties de Merlin permet de mettre en lumière différents langages politiques élaborés pour séduire un large auditoire. L'audience de ces prophéties va bien au-delà des milieux de cour et des hommes d'Église. Les textes, le plus souvent en latin, des versions savantes des prophéties de Merlin qui ont circulé en Grande-Bretagne, en France et en Europe du Sud, ne résument pas à eux seuls l'ampleur de la diffusion de certaines prophéties, car elles furent connues du peuple dans des adaptations orales, simplifications des versions latines, ou sous des formes antérieures aux versions écrites. Il est en effet utile de tenter d'appréhender comment les prédictions de Merlin étaient perçues par ceux qui n'avaient pas les compétences pour lire un texte et qui étaient ainsi exclus de la culture livresque, car ces prophéties relèvent, à l'origine, d'une tradition orale galloise, mise tardivement par écrit. Les interactions entre textes latins et rumeurs populaires dérivées de ces textes, ou provenant d'une tradition orale littéraire, révèlent une forme de communication politique entre les élites sociales et les anonymes, et laissent entrevoir l'étendue sociale de la transmission de prédictions qui, bien que très obscures, intriguaient le peuple. L'étude des rares textes historiques, littéraires et juridiques mentionnant la circulation de rumeurs prophétiques populaires de Merlin, permet de mieux comprendre comment la science merlinienne a pu séduire à la fois les élites et les anonymes éloignés de la culture savante, grâce à l'adaptabilité de langages politiques capables de se faire entendre de tous.

La littérature prophétique est un genre bien particulier, presque inclassable, dont la vocation originelle est de circuler oralement bien plus que sous la forme de compilation écrite. Le premier auteur qui mit les prophéties de Merlin par écrit, Geoffroy de Monmouth, explique clairement qu'il s'est décidé à les transcrire à la suite de bruits qui couraient sur la justesse de ces prédictions. En Angleterre aussi, elles sont donc connues oralement avant leur mise par écrit. Cependant, c'est d'abord un public de clercs qui en 
demande la transcription du gallois au latin. L'ampleur de la diffusion de la compilation de prophéties de Merlin rédigées par Geoffroy de Monmouth est incontestable, car plus de 70 manuscrits des prophéties et plus de 200 de l'Historia regum Britanniae à laquelle elles sont intégrées sont parvenus jusqu'à nous. Cependant, la possession de ces manuscrits latins, leurs traductions en langues vernaculaires ou leurs lectures orales, concernent essentiellement les clercs et certains nobles lettrés, et nous ne pouvons pas nous limiter aux seules traces écrites d'un genre littéraire dont la force réside dans sa propension à être oralisé. La prophétie n'a de sens, n'a de portée, que si elle circule selon le mode de la rumeur prophétique.

Mais c'est aussi parce qu'elles sont mises par écrit que les prophéties peuvent entrer dans le registre du savant et du sacré, et acquérir ainsi une légitimité durable dans une société où le savoir, comme les Révélations, passent par l'autorité de l'écrit. L'étude de l'audience des prophéties de Merlin peut nous permettre, à travers quelques exemples, de comprendre comment un langage politique peut s'adapter à plusieurs types d'auditoires et faire le lien entre les traditions orales et l'écrit savant.

Cette étude débutera par une présentation des origines orales des prophéties galloises de Merlin, qui circulent largement par la rumeur prophétique et qui intriguent tellement les érudits qu'elles font l'objet d'une mise par écrit au sein d'un corpus qui deviendra vite canonique. Puis nous verrons, en prenant l'exemple de l'Écosse, comment les rumeurs prophétiques peuvent être propagées en s'appuyant sur une source écrite qui fait autorité, assurant la propagation d'un double langage politique, à la fois savant et populaire. Nous analyserons ensuite l'efficacité plus complexe encore d'un langage politique merlinien exporté en France, à travers les textes des procès de Jeanne d'Arc, pour montrer comment un texte latin très hermétique peut se mêler à des croyances locales populaires pour réussir à convaincre un large auditoire. Et nous ne pourrons pas clore cet article sans dire quelques mots de l'Italie, terre des rumeurs prophétiques merliniennes dissidentes et seconde patrie des prophéties de Merlin, où nous verrons que les plus humbles pouvaient facilement accéder à la science de Merlin.

\section{Rumeurs prophétiques et tradition orale : un langage politique autochtone récupéré par le pouvoir anglais}

\section{Des prédictions issues de la tradition orale}

Les origines des prophéties de Merlin sont difficiles à situer dans le temps puisque longtemps elles ne circulent que par l'oralité. La littérature cymrique est très ancienne, et les plus anciens textes prophétiques remontent sans doute au $\mathrm{VI}^{\mathrm{e}}$ siècle avant J.-C., époque au cours de laquelle les Bretons perdent définitivement le pouvoir sur la plus grande partie de l'île de Bretagne 
et se réfugient au pays de Galles, en Cornouailles, et en Petite Bretagne continentale. Des poèmes prophétiques apparaissent alors dans le contexte de la lutte pour conserver une indépendance face aux occupants germaniques. Les prophéties de Merlin sont centrées sur le retour au pouvoir des Bretons qui s'uniront pour renverser les dominations étrangères et retrouver leur indépendance en régnant sur la totalité de l'île de Bretagne. La légende de Merlin s'est donc formée au cours des siècles qui suivirent la perte d'indépendance des Bretons. Les prophéties que Geoffroy de Monmouth attribue à Merlin sont certainement bien antérieures au XII ${ }^{\mathrm{e}}$ siècle, mais il est impossible de dater avec certitude cette compilation de prophéties nées du besoin de conserver une identité politique et un espoir breton. Toutefois, leur mise par écrit fut sans doute assez tardive, et les sources écrites galloises parvenues jusqu'à nous sont postérieures à Geoffroy de Monmouth. Au XII ${ }^{\mathrm{e}}$ siècle, Giraud de Cambrie dit avoir finalement découvert à Nefyn, après bien des recherches ${ }^{1}$, un manuscrit breton des prophéties de Merlin, manuscrit qu'il n'a sans doute jamais trouvé, mais dont l'autorité lui était nécessaire pour assurer la crédibilité des prédictions qu'il présentait ${ }^{2}$. Certes, les Gallois ne méconnaissaient sans doute pas la conservation écrite des prophéties, mais à l'époque leur transmission empruntait bien plus souvent la voie orale que la voie écrite.

Les érudits et les puissants gallois n'avaient rien à gagner à mettre systématiquement par écrit un corpus de prophéties qui circulaient déjà suffisamment de manière orale, avec l'avantage de ne pouvoir être analysées par l'ennemi qui demeurait dans la crainte de leur réalisation.

Mais après Geoffroy de Monmouth, lorsqu'un auteur cite une nouvelle prophétie de Merlin, il fait presque toujours référence au vieux livre de Merlin, dans lequel la prophétie aurait été retrouvée. L'écriture valide « un ensemble de croyances sous une forme canonique qui résiste au temps », selon la formulation de Jack Goody ${ }^{3}$. Les représentations de Merlin dans les manuscrits le montrent souvent en train de lire ou de dicter. Le livre de Merlin est cité de façon récurrente par les commentateurs de ses prédictions qui ont besoin d'une autorité écrite appartenant au passé pour décrire l'avenir.

Cependant, au $\mathrm{XII}^{\mathrm{e}}$ siècle, au moment où les clercs érudits découvrent les prophéties de Merlin par écrit, elles sont déjà connues depuis longtemps du peuple gallois qui n'a pas eu besoin d'attendre Geoffroy de Monmouth. C'est l'auteur lui-même qui nous explique qu'il ne fait que mettre par écrit les rumeurs qui circulent déjà oralement :

1. Giraud de CAmbrie, Giraldi Cambrensis opera, J. F. Dimock éd., vol. VI, Itinerarium Kambriae. Descriptio Kambriae, Londres, 1868, Livre II, chapitre VI, p. 124.

2. Geoffroy de Monmouth fait lui aussi référence à un vieux livre breton censé être l'unique source de l'Historia regum Britanniae.

3. JACK GoODY, Pouvoirs et savoirs de l'écrit, Paris, 2007. 
Je n'étais pas encore parvenu à ce point de mon histoire quand, au bruit qui se faisait autour du nom de Merlin, mes contemporains me poussèrent de tous côtés à publier ses prophéties, et parmi eux, principalement, Alexandre, l'évêque de Lincoln, un homme très pieux, d'une sagesse extrême ${ }^{4}$.

Ces rumeurs dérivent de la littérature orale qui est à l'origine conçue pour des puissants, et sont propagées par des bardes ayant une position sociale notoire. Martin Aurell rappelle la précocité de la littérature cymrique et l'importance des bardes luttant contre les invasions. Ces bardes faisaient partie de l'élite et pouvaient assumer des fonctions de guerrier ou de druide ${ }^{5}$. Mais comment alors ces prophéties pouvaient-elles arriver aux oreilles des gens du peuple ? Il existe différentes catégories de bardes, dont le barde en chef, barde royal (pencerdd), le barde de cour, ou, après la fin de l'indépendance galloise, le barde attaché à un seigneur (teuluwr), qui joue aussi le rôle politique de messager, en apportant des informations d'un chef à un autre. Mais il existe aussi des bardes itinérants (clerwr), qui sont plutôt des ménestrels itinérants. Ce sont des conteurs, des musiciens, mais aussi des amuseurs et des colporteurs de nouvelles. Certes, cette distinction est sans doute artificielle, comme le note Martin Aurell, car un même barde pouvait changer de statut en fonction de son âge et de son expérience. Toujours est-il que nombreux étaient les bardes ou ménestrels itinérants, surtout à la fin du $\mathrm{XIII}^{\mathrm{e}}$ siècle, après la perte d'indépendance du pays de Galles qui vit disparaître les bardes de cour. Ils colportent des prophéties politiques, des prophéties qui dérangent fortement le pouvoir anglais. Celui-ci légifère en effet régulièrement contre ceux qu'il nomme des vagabonds, des vauriens. Édouard $\mathrm{I}^{\mathrm{er}}$, après la défaite galloise, ordonne que les «Westres Barthes et Rymors » ne soient plus acceptés ni autorisés dans le pays, de peur que par leurs imprécations et leurs mensonges ils n'entraînent les gens à faire le mal, et ne s'imposent comme une charge sur le peuple ${ }^{6}$.

Henri IV craint lui aussi les ménestrels itinérants à l'époque de la révolte menée par le gallois Owen Glendower. Ainsi, en 1402, il ordonne:

Item, pur eschuir pluseurs diseases \& meschiefs qont advenuz devaunt ces heures en la terre de Gales par pluseurs westours rymours ministralx \& autres

4. Geoffroy de Monmouth, Histoire des rois de Bretagne, trad. L. Mathey Maille, Paris, 1992, p. 157. Texte latin : «Nondum autem ad hunc locum historie perueneram, cum de Merlino diuulgato rumore compellebant undique contemporanei mei prophetias ipsius edere : maxime autem Alexander Lincolniensis episcopus, uir summe religionis et prudentie.» (Geoffroy de Monmouth, Historia regum Britanniae, 1, Bern, Burgerbibliothek, MS. 568, N. WRIGHT éd., Cambridge, 1984, p. 73.)

5. M. Aurell, La légende du roi Arthur, Paris, 2007, p. 39-40.

6. Londres, British Museum, ms. Harleian 696, fo 82. Registrum vulgariter Nuncupatum, "The record of Caernarvon », H. ElLIS éd., Londres, 1939, p. 132 ; T. STEPHENS, The literature of the Kymry: being a Critical Essay on the History of the Language and Literature of Wales, Llandovery, 1849, p. 104 ; M. E. GRIFFITH, Early vaticination in Wales, with English parallels, Cardiff, 1917, p. 216. 
vacabondes ordeignez est \& establiz qe nul westour rymour ministrall ne vacabond soit aucunement sustenuz en la terre de Gales pur faire kymorthas ou coillage sur la commune people illoeqes ${ }^{7}$.

Les ordonnances contre les bardes et les ménestrels, assimilés à des vauriens, seront encore éditées sous Henri VIII puis sous Élisabeth ${ }^{\text {re }}$. S'ils sont craints, c'est que ces ménestrels itinérants colportent des rumeurs prophétiques qui incitent à la révolte. Les prédictions assurant le retour de la gloire bretonne sont le ciment de l'identité galloise.

\section{Langages politiques et échanges linguistiques}

Le peuple gallois pouvait donc aisément accéder aux prophéties de Merlin grâce notamment aux ménestrels itinérants. Mais comment appréhender la diffusion de prophéties auprès de ceux dont on ne parle pas ? Pour trouver les sources faisant état de prophéties de Merlin connues explicitement par des anonymes, il faut attendre le XII ${ }^{\mathrm{e}}$ siècle et se tourner vers des sources littéraires. Ainsi, Giraud de Cambrie transcrit des prophéties orales. Il raconte comment le roi Henri II se fait interpeller par une Galloise qui lui annonce sa mort prochaine sur une pierre surnaturelle, pierre qui servait de pont sur une rivière. Henri rentre d'Irlande et passe par Saint-David, au pays de Galles :

Comme le cortège s'avançait, le clergé marchant en file selon les rites, une femme galloise s'est jetée aux pieds du roi pour se plaindre de l'Évêque de Saint David's. Ses propos furent expliqués au roi par un interprète. Rien ne pourrait être fait séance tenante de sa requête, aussi elle gesticula violemment avec ses mains et lorsque tout le monde écoutait, eut l'impudence de crier d'une voix forte: "Venge nous maintenant Lechlavar! Venge le peuple gallois tout entier sur cet homme ! » Elle fut retenue puis éloignée de là par ceux qui comprenaient la langue galloise. En s'éloignant elle cria de plus en plus fort. Elle faisait allusion à cette fiction et prophétie de Merlin, bien connue, qu'un roi d'Angleterre qui viendrait tout juste de conquérir l'Irlande, et qui aurait été blessé dans ce pays par un homme à la main rouge, mourrait de retour à Saint David's en passant sur Lechlavar ${ }^{8}$.

7. Londres, Archives Nationales, Stat. IV. Henri IV, C. 27. I. BowEN, The statutes of Wales, Londres, 1908, p. 34. "De la même façon, pour éviter de nombreux inconvénients et troubles survenus autrefois au pays de Galles du fait de nombreux jongleurs, rimeurs, ménestrels et autres vagabonds, il est ordonné et décidé qu'aucun jongleur, rimeur, ménestrel ou vagabond ne soit en quelque façon gardé au pays de Galles pour y recueillir de l'argent auprès des gens du peuple au nom d'une coutume ou d'une redevance. »

8. Texte latin: «Accidit autem ut procedente seriatim processione et rite precedente, mulier Kambrica ad pedes regis se subito provolveret ; quae et, querimonia de loci illius episcopo facta et regi per interpretis linguam exposita, quoniam ius suum incontinenti non est assecuta, voce altisona et proterva cum manuum quoque complosione non modica, cepit coram omnibus exclamando ingeminare: "Vindica nos hodie, Lechlavar, vindica genus et gentem de homine hoc". Cumque ab illis qui Britannicam linguam noverant inhiberetur et expelleretur, ipsa quoque tanto fortius et acrius in hunc modum vociferabat; alludens illi ficticio vulgari, nec vero Merlini proverbio, quo dici solebat Anglie regem Hibernie triumphatorem, ab homine cum rubra manu in Hibernia vulneratum, per Meneviam redeundo super Lechlavar moriturum. » 
Henri II connaît la prophétie. Il franchit la pierre et se permet de traiter Merlin de menteur :

Le roi, parce qu'il avait d'aventure entendu la prophétie, s'approcha de la pierre, s'arrêta brièvement devant elle, puis, la fixant d'un œil sérieux, la franchit hardiment; ensuite, se retournant et regardant la pierre, il fulmina ainsi contre le prophète : « Merlin est un menteur. Qui le croira maintenant ${ }^{9}$ ?»

Un homme qui se trouvait parmi la foule, nous dit Giraud, entendit la remarque du roi et lui rétorqua qu'il n'était pas le roi conquérant de l'Irlande dont parlait Merlin : «Tu n'es pas le roi qui doit conquérir l'Irlande et Merlin n'a pas parlé du tout de toi ${ }^{10}$.»

La Galloise s'exprime dans sa langue, et son interpellation nécessite l'intervention d'un interprète. Déçue par le roi, elle prend le risque de parier sur une proche vengeance. Elle connaît bien les prophéties de Merlin qui portent les espoirs de tout son peuple. La communication linguistique a son importance dans la situation politique que connaît alors la Grande-Bretagne. Les peuples parlent soit des langues celtiques soit l'anglais, alors que le roi d'Angleterre et l'aristocratie gouvernante qui ambitionnent de dominer la Grande-Bretagne parlent français, et l'administration utilise le latin. Les difficultés de communication sont donc importantes. Pourtant la prophétie entraîne des échanges linguistiques véhéments entre puissants et anonymes qui ne parlent pas la même langue.

Les traditions locales qui se rattachent à cette prophétie font que les Gallois devaient la connaître et la comprendre sans avoir besoin de médiation. Giraud nous la présente comme une prophétie populaire. Mais pour que le roi et l'aristocratie comprennent ces prophéties il fallait les traduire. Cette prophétie est cependant tellement répandue que le roi Henri la connaît bien. Pourtant Giraud est vraisemblablement le premier à la mettre par écrit. Avec ce texte, nous avons un exemple des prophéties galloises bardiques assimilées par le peuple et littéralement traduites du gallois oral vers le latin écrit. Cette prophétie reprend des thèmes chers à la prophétie galloise, comme l'homme à la main rouge du sang ennemi, une figure récurrente du héros des prophéties galloises comme en témoignera la popularité du gallois Owain Lawgoch au XIV ${ }^{\mathrm{e}}$ siècle, Owain « la main rouge ». L'exemple de la prophétie

L'intégralité du récit sur la prophétie de Lechlavar figure dans l'Itinerarium Kambriae. Ce récit figure aussi dans l'Expugnatio Hibernica, mais sans l'ultime remarque du défenseur de Merlin, ajoutée dans l'Itinerarium Kambriae (GIRAUD DE CAMBRIE, Itinerarium Kambriae, J.-F. DiMOCK éd., op. cit., p. 108).

9. Texte latin : «Accedens igitur ad lapidem rex, quia forte vani illius vaticinii mentionem audierat, ad pedem lapidis paulisper gradum sistens et eundem acriter intuens, incunctante tamen passu audacter pertransiit. Verso itaque vultu ad lapidem respiciens, in vatem invectus, verbum hoc indignanter emisit : Merlino mendaci quis de cetero fidem habeat ? ( GIRAUD DE CAMBRIE, Itinerarium Kambriae. J.-F. Dімоск éd., op. cit., p. 109.)

10. « Tu vero non es rex ille, qui Hiberniam conquirere debet ; nec de te Merlinus mentionem fecit. », ibid., p. 109. 
de Lechlavar montre la force de l'oralité dans la diffusion d'un texte, avant sa mise par écrit. Giraud de Cambrie théâtralise la mise en scène de cette altercation pour montrer le courage d'Henri II. Le roi semble même passer près de la pierre Lechlavar pour provoquer les Gallois qui attendent sa mort avec impatience. C'est dire l'attention qu'il accorde à cette vaticination populaire que Giraud de Cambrie est le premier à traduire en latin.

\section{Prophéties savantes et rumeurs prophétiques : un double langage politique}

La prophétie de Lechlavar est loin d'être la dernière prédiction qui circule dans le contexte des affrontements entre les peuples d'origines celtiques et les Anglo-Normands. Les exemples de prophéties galloises politiques circulant oralement se multiplient lorsque, sous Édouard ${ }^{\mathrm{er}}$, le conflit entre Gallois et Anglo-Normands atteint son paroxysme ${ }^{11}$. Mais à la fin du $\mathrm{XIII}^{\mathrm{e}}$ siècle, les prophéties les plus déterminantes dans les conflits armés opposant les Anglais aux Gallois ou aux Écossais, ont toutes un équivalent écrit latin, le plus souvent en vers. Nous sommes donc passés à une société où l'écrit valide systématiquement les croyances, qu'elles soient populaires, élitistes, ou qu'elles mêlent les deux types de publics, montrant ainsi que le pouvoir a bien compris la nécessité de s'emparer de toutes les prophéties, même de celles qui lui nuisent, pour les analyser et en tirer profit en modifiant leur sens.

\section{Une rumeur inquiétante diffusée par des prêcheurs}

Pour s'en convaincre, il faut nous arrêter sur un exemple de diffusion attestant la propagation de rumeurs prophétiques dont la véracité repose sur l'existence d'un texte latin. Nous sommes toujours dans le contexte d'une guerre d'indépendance. Édouard $\mathrm{I}^{\mathrm{er}}$ a vaincu les Gallois, mais cette fois, ce sont les Écossais qui reprennent le flambeau de l'espoir breton. Une nouvelle prophétie, absente de la compilation de Geoffroy, relate la mort du roi anglais. Nous en trouvons la trace dans une lettre adressée à un officiel anglais. Ce courrier est rédigé par un lord écossais pro-anglais ${ }^{12}$ soucieux d'informer les autorités anglaises sur la situation de l'Écosse. Il fait état de la dangereuse prophétie du roi cupide, qui trouble les esprits en laissant entendre qu'Édouard $\mathrm{I}^{\mathrm{er}}$ va mourir et laisser place au nouveau règne des Bretons et de leurs cousins Écossais redevenus maîtres de l'île de Bretagne.

11. C. DanIEL, Les prophéties de Merlin et la culture politique (XII ${ }^{e}-\mathrm{XVI}^{e}$ s.), Turhnhout, 2007, p. 181-218; EAD., «Les prophéties de Merlin et l'impérialisme anglais », dans Actes du $22^{e}$ congrès arthurien de Rennes [en ligne], site de la Société internationale arthurienne, http://www.uhb.fr/alc/ias.

12. Selon G. BARRow, le rédacteur de cette lettre pourrait être Alexander Abernethy. G. BARROw, Robert Bruce and the community of the realm Scotland, Édimbourg, 1988, p. 172-173. 
La lettre date du 15 mai 1307, soit moins de deux mois avant la mort effective du roi Édouard, non loin de la frontière écossaise.

[...] Dieux si ly plest, teyne la vie nostre Seignur le Roy ; kar a quel oure qe nous failum de luy, qe Dieux defend, il dient apertement qe tous serrent de une part, ou il lor couent morer ou voyder la terre et tous ceus qe eyment le Roy, si autre counsail et eyde ne soyt mys entre eux. Kar les prechours les funt entendre qil ount trove vers de Merlin, coment après la mort le Roi Coueytous les gentz Descose et les Brutouns qil entendent par les Galeis se aerderent ensemble, et averent la sovereine mayn et lor volunte, et viverunt ensemble de un accord iekes au fin du monde ${ }^{13}$.

Ce document est un exemple très parlant du mode de propagation des rumeurs prophétiques politiques au XIV ${ }^{\mathrm{e}}$ siècle. Elles circulent par l'intermédiaire de prêcheurs écossais, probablement des franciscains ${ }^{14}$, dont on connaît le ralliement au leader écossais Robert Bruce, et qui suivaient son armée pour exhorter le peuple à défendre le royaume. Ces franciscains utilisaient Merlin pour faire de Bruce le sauveur des Écossais et de l'île de Bretagne.

Contrairement à la prophétie sur la mort d'Henri II, il existe plusieurs versions écrites très élaborées de la prédiction sur la mort d'Édouard ${ }^{\mathrm{er}}$. Il s'agit d'une prophétie dérivée de celle de l'espoir breton sur l'union des peuples celtiques, à laquelle a été ajoutée la mort d'un roi cupide. L'informateur qui rédige la lettre explique que les prêcheurs ont découvert une vaticination de Merlin sur le roi cupide, trouvée de toute évidence dans un livre. La prophétie convainc le peuple parce qu'elle est écrite dans un livre de Merlin. La mort d'Édouard ${ }^{\mathrm{er}}$ concrétisera la première partie de la prophétie, ce qui lui vaudra une importante postérité. Chacun attendait donc le moment où la seconde partie de la prophétie, sur l'union de l'île de Bretagne, allait fatalement devenir réalité.

\section{L'autorité de l'écrit prophétique}

Les vers de Merlin dont parle le lord dans sa lettre sont une prophétie latine exacerbant le racisme anti-anglais et glorifiant les alliances des peuples celtiques contre les Saxons et leurs descendants. Sous sa forme latine, la version écrite de ce poème, que nous pouvons nommer Regnum Scottorum, n'est pas très ancienne et remonte sans doute au XIII ${ }^{\mathrm{e}}$ siècle. Le poème fait allusion à l'interrègne après la mort d'Alexandre III d'Écosse, et explique comment, selon Merlin et la Sibylle, l'Écosse retrouvera sa liberté après la

13. Calendar of documents relating to Scotland preserved in Her Majesty's Public Record Office, J. BAIN éd., Londres, 1881, t. 2, p. 537.

14. N. Gallagher, "The Franciscans and the Scottish wars of independence: an Irish perspective », Journal of Medieval History, t. 32, 2006, p. 3-17. 
mort du roi d'Angleterre. En voici un extrait, caractéristique de la violence anti-anglaise ${ }^{15}$ inhérente au poème :

Sanguine Saxonico tincta rubebit humus

Flumina manabunt, hostili tincta cruore

Perfida gens omni lite subacta ruet.

Regnabunt Britones, Albani gentis amici ;

Antiquum nomen insula tota feret $[\ldots]$

Cum Scotis Britones regna paterna regent

Regnabunt pariter, in prosperitate quieta.

Hostibus explusis, judicis usque diem.

Hystorie veteris Gildas luculentus orator,

Quem retulit, paruo carmine plura notans ${ }^{16}$.

Cette prédiction est très populaire, et nous la retrouvons chez des chroniqueurs écossais, mais aussi chez des chroniqueurs anglais ${ }^{17}$. On la retrouve aussi très souvent dans des manuscrits comportant des compilations anglaises de prophéties latines ${ }^{18}$ ou simplement quelques prophéties isolées, mais aussi dans des papiers d'État ${ }^{19}$. Ainsi la prophétie est-elle vraiment sortie du cadre littéraire pour investir les documents officiels et la correspondance d'État, ce qui atteste son rôle politique. Cependant, elle est également diffusée sur le mode de la rumeur relayée par les prédicateurs, et se glisse sans doute aussi dans le texte de chansons populaires. L'efficacité de ces prophéties sur l'union de la Bretagne est incontestable. Elles sont tellement crédibles que l'Angleterre s'en empare, récupérant l'idée d'une union pro-

15. Les Anglais sont désignés sous le nom de Saxons. Les Gallois sont nommés Bretons et les Écossais sont ceux de la race d'Alban.

16. W. SKENE, Chronicles of the Picts, chronicles of the Scots, and other early memorials of Scottish history, Édimbourg, 1867, p. 118. Traduction : "La terre rougira, colorée du sang des Saxons. Les fleuves couleront, teintés du sang des ennemis. La race perfide, toute querelle anéantie, s'écroulera. Les Bretons règneront, amis de la race d'Alban ; l'île toute entière portera l'ancien nom [...]. Les Bretons, avec les Écossais, gouverneront les royaumes de leurs pères. Ils règneront ensemble, dans la prospérité et la paix, ayant bouté leurs ennemis dehors, jusqu'au Jugement dernier. Dans son Histoire d'autrefois, le merveilleux prédicateur Gildas a raconté ce jour, consignant en une brève formule bien des événements. »

17. WALTER DE COVENTRY, The Historical collections of Walter of Coventry, edited from the ms. In the library of Corpus Cristi college, Cambridge, W. STuBbS éd., 2 vol., Londres, 1872-1873, (RS 58I), p. 25-26. The Chronicle of Peter of Langtoft, T. WRIGHT éd., 2 vol., Londres, 1866, (RS 47II), p. 448-449. Walter Bower, Scotichronicon, in latin and English, D. E. R. WATt éd., 9 vol., Aberdeen, 1987, vol. II, Livre III, chap. 23, p. 60. Extracta e variis Cronicis Scocie, from the auncient manuscript in the advocates library at Edinburgh, Édimbourg, 1842, p. 26-27.

18. Nous citerons, entre autres manuscrits, les suivants : Londres, College of Arms, ms. Arundel XXX (fin du XIII ${ }^{e}$ s.). Londres, British Library, ms. Cotton Titus D. VII ( $\mathrm{XIV}^{\mathrm{e}} \mathrm{s}$.). Londres, British Library, ms. Cotton Claudius B. VII (XIV s.). Londres, British Library, ms. Cotton Vespasian E. VII (XV s.). Oxford, Bodleian Library, ms. Rawlinson D 248 ( $\mathrm{XV}^{\mathrm{e}}$ s.). Cambridge, Gonville and Caius College, ms. 249/277 (Xve s.). Dublin, Trinity College, ms. 516 (Xve s.).

19. London Public Record Office, ms. E 164/9. Le manuscrit date de 1291, mais les prophéties qu'il contient ont été ajoutées aux $\mathrm{XIV}^{\mathrm{e}}$ et $\mathrm{XV}^{\mathrm{e}}$ siècles. 
chaine de l'île de Bretagne en jouant sur les multiples interprétations de la prédiction ${ }^{20}$.

Néanmoins, ces prophéties exacerbent aussi la haine ressentie par les Anglais, une haine très vive, comme en témoignent les chansons populaires violemment anti-écossaises rapportées à la fin de la chronique de Pierre de Langtoft, biographe d'Édouard I ${ }^{\text {er }}{ }^{21}$. Il ne s'agit pas simplement d'une guerre des chefs, mais bien d'un conflit entre deux peuples qui sont en train de devenir des nations. Les versions savantes ou littéraires des prophéties ont un équivalent oral qui leur donne une force de persuasion pour s'assurer l'adhésion populaire à des guerres censées ramener l'île de Bretagne au temps de son âge d'or.

\section{Rumeurs prophétiques, textes savants et croyances populaires}

Éloignons-nous maintenant de l'île de Bretagne pour voir comment le langage politique que constituent les prophéties de Merlin a pu s'exporter en France, ennemie de l'Angleterre, et trouver grâce aux yeux des Français, quel que soit leur niveau de culture.

\section{Jeanne d'Arc, la fille du Bois Chesnu}

S'il est aisé de comprendre comment, en terre celtique, la tradition orale favorise la circulation de rumeurs prophétiques dans un pays où la littérature est tournée vers les promesses d'un retour du pouvoir breton, il est plus difficile de comprendre comment les prophéties ont pu être popularisées en France pendant la guerre de Cent Ans. Les prophéties concernant la France sont assez rares avant le début de cette guerre. Pourtant, les Français adoptèrent facilement le Merlin des Anglais, car ses prédictions, volontairement obscures et malléables, constituent un langage politique capable de s'adapter à différentes situations politiques. Alors que les Anglais retournèrent le sens des présages du Merlin des Gallois, les Français surent utiliser le Merlin des Anglais.

L'exemple choisi ici est celui des rumeurs sur Jeanne d'Arc, attestées lors de ses procès. Nous sommes toujours dans le contexte des guerres pour l'indépendance, propices à la circulation des prophéties de Merlin. Des prophéties circulaient sur la venue d'une pucelle qui sauverait la France, avant que Jeanne ne vienne vers le roi. Deux prophéties de Merlin vont donc très vite lui être associées et assurer sa popularité. L'une fait d'elle la fille du Bois Chesnu, l'autre en fait une Vierge zodiacale qui vaincrait le Sagittaire symbolisant les archers anglais. Cette seconde prophétie, tirée comme la pre-

20. L. A. Cооте, Prophecy and public affairs in later Medieval England, Woodbridge, 2000 , p. 72.

21. Voir l'édition critique de J.-C. ThioLIER, Le règne d'Édouard I ${ }^{e r}$, Créteil, 1989. 
mière de l'Historia regum Britanniae, semble un peu moins populaire, car il s'agit d'une prophétie astrologique plus difficile à saisir. Toutefois, les deux prophéties ont parfois été associées ${ }^{22}$.

Colette Beaune rappelle que la circulation des prophéties « dépassait [...] les milieux cléricaux et le cénacle des hommes au pouvoir. Toute prophétie [...] avait sa version en français. Eustache Deschamps ou Christine de Pisan en faisaient des ballades ${ }^{23}$ ». Jeanne pouvait donc avoir connaissance des versions orales des prophéties savantes de Merlin.

Lors de son procès en condamnation, Jeanne doit s'expliquer en particulier sur une prophétie de Merlin qui la concerne et qui a fait beaucoup de bruit. Elle nous livre un aspect folklorique du contexte de diffusion de cette prophétie devenue très populaire :

Item dit bien qu'il y a ung bosc que on appelle le Bosc Chesnu, que on voit de l'huys de son pere; et y a petite espace non pas d'une lieue; mais qu'elle ne sçait ne ouyt oncques dire que les fees y repperassent.

Item dit qu'elle a ouy dire a son frere que on disoit au pays qu'elle avoit prins ses revelacions a l'arbre et es fees. Mais non avoit, et luy disoit bien le contraire. Et dit oultre, quand elle vint devers le roy, que aulcuns demandoyent si en son pays avoit point de boys que on appelast le Boys Chesnu ; car il y avoit prophecies qui disoyent que de devers le Boys Chanu debvoit venir une pucelle qui venoit faire merveilles ; mais en ce n'a point adjousté de foy ${ }^{24}$.

C'est par la rumeur que Jeanne a eu accès à cette prophétie. Ce Bois Chesnu est un lieu rattaché à des croyances, des superstitions populaires sur un Arbre aux Fées. Il intrigue fortement les interrogateurs de Jeanne.

Item interroguee de l'arbre :

Respond que assez prez de Dompremy [est] ung arbre qui se appelle l'Arbre des Dames ; et les aultres l'appellent l'Arbre des Fees ; et auprez a une fontaine ; et a ouy dire que les gens malades des fiebvrez en boyvent; et mesmes en a veu aller querir pour en guarir. Mais ne sçait se ilz en garissoyent ou non. $[\ldots]^{25}$.

La prophétie est rattachée à un folklore et à des superstitions populaires qui renforcent son discrédit aux yeux des théologiens. Jeanne est la fille du Bois Chesnu, un bois où les fées ont élu domicile et où se trouve une fontaine guérisseuse.

22. Dunois rapporte dans sa déposition que des vers annonçant la venue d'une pucelle du Bois Chesnu qui chevaucherait sur le dos des archers furent transmis à Suffolk, alors prisonnier. Procès de condamnation et de réhabilitation de Jeanne d'Arc dite la Pucelle, J. QUICHERAT éd., t. 3, Paris, 1845 , p. 15.

23. C. Beaune, Jeanne d'Arc, Paris, 2004, p. 111.

24. Procès de condamnation de Jeanne d'Arc, P. Tisset éd., t. 1, Paris, 1970, p. 67.

25. Ibid., p. 65. 


\section{La source savante d'une prédiction populaire}

La prédiction ressemble à une prophétie issue du folklore populaire. Mais la situation est bien plus complexe, car cette prophétie n'est rien d'autre que la version populaire d'une prophétie savante, que Jean Bréhal, Grand Inquisiteur de la foi en France, prendra la peine de disséquer longuement pour défendre Jeanne dans la Recollectio du procès en réhabilitation ${ }^{26}$.

Reportons nous aux écrits de Jean Bréhal, qui, avant d'analyser la prédiction savante, en rapporte la popularité :

À ce sujet une ancienne rumeur populaire se répandit qu'une jeune fille devait naître de ce lieu qui accomplirait des merveilles, selon ce qu'on rapporte dans le procès. Une importante confirmation est apportée à cela par le Roman de Brut dans lequel on lit que le devin anglais Merlin avait prédit ainsi : « Du bois chenu sortira une pucelle qui portera remède au mal ${ }^{27}$ ».

Jean Bréhal résume en quelques mots la version orale de la prophétie qui devient très claire. L'écrit vient toujours valider la rumeur prophétique, et Bréhal cite une source qui n'est peut-être qu'une version du Brut de Wace avec les prophéties de Merlin interpolées. Cependant, selon l'Inquisiteur, cette prédiction circulait oralement de toute antiquité.

Si l'on considère la version savante de la prophétie issue de la compilation de Geoffroy de Monmouth, on peut constater qu'elle est en phase avec la prophétie populaire et les croyances qui l'entourent. Il y est question de trois sources, l'une bénéfique, les autres maléfiques, ces dernières étant asséchées par une jeune fille de la Ville-du-Bois-de-Canut :

Dans la ville de Winchester, trois sources jailliront, dont les ruisseaux diviseront l'île en trois parties. Qui s'abreuvera à l'un jouira d'une très longue vie sans être accablé par la maladie. Qui s'abreuvera à l'autre mourra d'une faim insatiable et portera sur son visage la pâleur et l'effroi. Qui s'abreuvera au troisième disparaîtra subitement et son corps ne pourra être enseveli.[...] Une jeune fille de la Ville-du-Bois-de-Canut sera envoyée sur place pour remédier à ce phénomène. Initiée à toutes les connaissances c'est de son seul souffle qu'elle assèchera les sources nuisibles ${ }^{28}$.

26. Procès en nullité de la condamnation de Jeanne d'Arc, P. Duparc éd., tome 2, Paris, 1979, p. 471-473. L'instruction du procès en réhabilitation est confiée à Jean Bréhal.

27. Texte latin: «De quo vulgaris et antiqua percrebuit fama, puellam unam ex eo loco debere nasci, que magnalia faceret, prout etiam in processu refertur. Ad quod videtur non parum suffragari id quod in historia Bruti legitur, Merlinum vatem anglicum sic predixisse: "Ex nemore Canuto puella eliminabitur, ut medele curam adhibeat." » (Ibid., p. 412.)

28. Geoffroy de Monmouth, trad. L. Mathey Maille, op. cit., p. 165. Texte latin : « Tres fontes in urbe Guintonia erumpent quorum riuuli insulam in tres portiones secabunt. Qui bibet de uno diuturniori vita fruetur nec superuenienti languore grauabitur. Qui bibet de altero indeficienti fame peribit et in facie ipsius pallor et horror sedebit. Qui bibet de tertio subita morte periclitabitur nec corpus ipsius subire poterit sepulchrum. [...] Ad hec ex urbe canuti nemoris eliminabitur puella ut medele curam adhibeat. Que ut omnes artes inierit, solo anhelitu suo fontes nociuos siccabit. »(GEOFFroy DE MONMOUTH, op. cit., p. 78.) 
La Ville-du-Bois-de-Canut est devenue le Bois Chesnu, et la prédiction est simplifiée pour ne retenir que le récit d'une jeune fille venue porter remède à un mal, en l'occurrence l'occupation anglaise. Pourtant, l'adaptation populaire n'est pas une vulgaire simplification de la prophétie savante. En effet, comment ne pas mettre en relation la source bénéfique de la prophétie avec la source guérisseuse du Bois Chesnu ? La prophétie populaire et le contexte dans laquelle elle s'intègre sont assez fidèles à la prédiction latine, mais l'interprétation populaire qui en est faite est finalement beaucoup plus convaincante que l'obscure prédiction écrite. La fille du Bois Chesnu, domaine des fées et des fontaines guérisseuses, accomplira des merveilles qui sauveront la France.

La fin de la prophétie savante parle des malheurs d'une nation assimilée à l'Angleterre, le texte étant difficilement applicable à la France. Jean Bréhal l'analysera pourtant mot à mot pour en montrer la cohérence, faisant ainsi preuve d'une grande ingéniosité.

Il existait sans doute une version orale populaire plus étoffée que celle des rumeurs citées dans les procès de Jeanne. Reste à savoir comment la prophétie savante a pu si bien s'adapter aux croyances populaires. Les propagandistes du roi Charles VII ont su extraire du corpus des prophéties de Merlin les deux seules dont l'hérö̈ne est une femme. La Vierge étant la patronne de la France, il était naturel que le salut du royaume vienne d'une vierge $^{29}$. Mais pourquoi Bréhal prétend-il que la rumeur populaire était fort ancienne ? Est-ce simplement pour lui donner plus de crédit? La prophétie de Merlin a-t-elle pu être connue du peuple avant que ne s'en mêlent les propagandistes de Charles VII ? C'est peu probable, mais il existait des croyances populaires sur un Bois Chesnu, croyances qui ont été assimilées à la prophétie de Merlin. Car, à bien lire Jean Bréhal, c'est peut-être ainsi qu'ont procédé les propagandistes pour diffuser la rumeur: ils sont allés chercher, dans Le livre de Merlin, une confirmation de la rumeur populaire, peut-être ancienne, sur une hérö̈ne issue d'un Bois Chesnu. Ils ont ensuite diffusé une version orale, proche de la version savante, pour attester que Jeanne était l'élue dont parlent le devin et les rumeurs populaires.

Personne n'a retrouvé le texte intégral de la version populaire qui circulait sur Jeanne, sans doute parce que ce texte n'existe pas. La véritable prophétie, celle qui fait autorité, se trouve dans Le livre de Merlin, ou dans un livre contenant les prophéties de Merlin, comme le Roman de Brut interpolé dont nous parle Bréhal. Il était utile de la simplifier oralement pour la rendre intelligible, mais pas d'en réécrire une version forcément considérée comme erronée.

L'exemple de cette prophétie de Merlin sur Jeanne est particulièrement intéressant pour l'étude des langages politiques accessibles à un large auditoire, car non seulement nous sommes en présence d'une prédiction 
savante rendue accessible au peuple par une assimilation à des superstitions populaires, mais par la suite la version orale est consignée dans les textes d'un procès pour mieux accuser l'héroïne de la prédiction, avant que l'Inquisiteur Jean Bréhal ne retourne à la version savante, latine, pour en prouver la légitimité en la commentant minutieusement lors du procès de réhabilitation ${ }^{30}$. Il y a donc des interactions complexes entre les langages politiques savant et populaire. C'est l'interprétation qui fait la force de la prophétie, autant que son adaptation aux croyances locales, le texte latin s'avérant manifestement inadapté pour une utilisation dans le royaume de France. La prophétie réussit ainsi à convaincre toutes les catégories sociales, et à marquer les esprits au point de figurer à maintes reprises dans les comptes rendus des procès de Jeanne.

\section{Sagesse populaire et texte savant}

Avec l'exemple des rumeurs sur Jeanne, nous voyons que les croyances populaires peuvent conforter une prophétie savante et lui donner ainsi une portée plus grande. Cependant, celui qui valide la prophétie, celui qui a le dernier mot dans l'analyse de la prophétie lors du procès de réhabilitation, Jean Bréhal, est un homme d'Église, et non des moindres. Nous allons voir dans un dernier exemple que parfois les prophéties de Merlin sont si bien intégrées à la culture populaire que ce sont les gens du peuple qui, mieux que les clercs, interprètent les versions savantes.

\section{Le contexte des rumeurs néo-joachimites}

La diffusion des prophéties en direction des couches populaires est frappante en Italie, dans les communes du Nord qui, au XIII ${ }^{\mathrm{e}}$ siècle, sont très sensibles aux arguments prophétiques. Parmi les innombrables prophéties de Merlin qui circulaient alors, certaines nous sont parvenues, mais peu sont déchiffrables complètement tellement elles sont liées à des contextes et des situations locales qui nous échappent. Bien davantage qu'en France, la péninsule italique a été le terrain d'expression du langage prophétique merlinien. C'est d'ailleurs en Italie qu'est née la seconde compilation de prophéties de Merlin, rédigée en français, et affranchie du texte de Geoffroy, celle attribuée à Maistre Richard d'Irlande ${ }^{31}$, un auteur franciscain joachimite se faisant passer pour un gibelin convaincu. Le mode privilégié de diffusion de ces prophéties en Italie demeure celui de la prédication. Salimbene de Adam, chroniqueur et prédicateur franciscain, qui fut longtemps joachimite, fait état

30. Pour l'analyse du commentaire de Bréhal, voir C. DANIEL, « Les prophéties de Merlin aux procès de Jeanne d'Arc », dans Littérature et droit du Moyen Âge à la période baroque : le procès exemplaire, Actes de la journée d'études du groupe de recherche Traditions Antiques et Modernités de Paris VII, S. Geonget, B. Meniel éd., Paris, 2008, p. 193-215.

31. Les prophecies de Merlin, L. A. PAton éd., 2 vol., New York et Londres, 1926. 
de l'importance des rumeurs merliniennes. Il rapporte que la validité des prédictions de Merlin faisait l'objet de nombreuses discussions entre prédicateurs ${ }^{32}$, et que ces prédictions, en particulier celles sur la survie de Frédéric II ${ }^{33}$, étaient très populaires en Italie. Le devin est devenu une autorité indispensable aux joachimites, parce que Merlin, plus qu'aucune autre figure prophétique, est capable de séduire un large auditoire. Sinon, pourquoi aller chercher le devin des Anglais pour parler des conflits entre cités, de Frédéric II et de la fin du Monde?

\section{Merlin interprété par un illettré}

Les prophéties joachimites étaient écoutées chez les petites gens et par là même l'autorité de Merlin reconnue des humbles. Dans ce contexte, les citoyens, même les plus modestes, veulent connaître leur sort et écoutent les anciennes paroles du mage celtique. Dans la cité de Parme, un cordonnier, Benvenuto, surnommé l'Asdente, est une célébrité locale car il a le don d'interpréter l'avenir. Il est connu pour avoir annoncé, entres autres événements, la mort du pape Nicolas III et l'élection de Martin IV. Pour lire dans le futur, Benvenuto s'appuie sur les prophètes bibliques, mais aussi sur la Sibylle, Joachim de Flore et bien entendu Merlin ${ }^{34}$. Alberto Milioli et Salimbene de Adam ont chacun rapporté l'audience de ce cordonnier auprès du peuple comme des puissants.

En ce temps là vivait dans la cité de Parme un certain pauvre homme, un cordonnier (qui fabriquait des sandales), un homme pur, simple et craignant Dieu. Il était courtois, c'est-à-dire qu'il avait de bonnes manières, et bien qu'il fut illettré, il avait un esprit éclairé, parce qu'il pouvait comprendre les écrits de ceux qui prédisaient le futur, comme l'abbé Joachim, Merlin, Méthode, les Sibylles, Isaïe, Jérémie, Josué, Daniel, l'Apocalypse et Michel Scot, l'astrologue de Frédéric II ${ }^{35}$.

Merlin est connu de ce prophète illitteratus, qui peut malgré tout interpréter les écrits des prophètes. Un peu plus loin ${ }^{36}$ Salimbene nous en dit davantage sur sa façon de travailler. Il écoute la lecture de passages des

32. Salimbene de Adam, Cronica, G. Scalia éd., t. 1, Turnhout, 1998, p. 367. Notons que l'Asdente utilise aussi la science de Michel Scot, astrologue de l'empereur Frédéric II, ce dernier étant l'anti-héros ou le sauveur de nombre de prophéties de Merlin.

33. C. DANIEL, Les prophéties de Merlin et la culture politique (XII ${ }^{e}-\mathrm{XVI}^{e}$ s.), op. cit., p. $349-363$.

34. Voir L'Enfer de Dante (XX, 118).

35. Texte latin : «Item his diebus erat in civitate Parmensi quidam pauper homo operans de opere cerdonico (faciebat enim subtellares), purus et simplex ac timens Deum et curialis, id est urbanitatem habens, et illitteratus, sed illuminatum valde intellectum habebat, in tantum ut intelligeret scripturas illorum qui de futuris predixerunt, scilicet abbatis Ioachim, Merlini, Methodii et Sibille, Ysaie, Ieremie, Osee, Danielis et Apocalipsis nec non et Michaelis Scoti, qui fuit astrologus Friderici secundi imperatoris condam. »(SALIMBENE DE ADAM, op. cit., p. 776.) 36. Ibid., p. 796. 
écrits de Merlin ou de Joachim, qu'il semble connaître par cœur, et sait les décrypter. Cet homme, nous dit l'auteur, est un prophète seulement dans le sens où il a un don pour comprendre les écrits de Merlin, de la Sibylle, de Joachim et d'autres qui ont prédit l'avenir, et si quelqu'un au cours de la lecture à voix haute de textes prophétiques omet un passage, il s'en rend compte immédiatement. Il demeure cependant un homme sans orgueil et sans vanité.

Qui dit prophète humble et populaire dit auditoire populaire, même s'il est évident que les puissants instrumentalisent le prophète cordonnier. Mieux que les prédicateurs, il pouvait se faire comprendre des siens et leur expliquer les prophéties de Merlin. Certes, il n'est pas surprenant que lorsque Dieu cherche un prophète, il s'adresse plutôt à quelqu'un qui n'a habituellement pas la parole. Mais ici le cordonnier n'est pas vraiment un prophète. Il comprend simplement les écrits des prophètes et sait les expliquer, ce qui constitue un renversement de l'autorité habituelle des clercs sur les textes, évidemment mieux lotis que les cordonniers pour les comprendre. L'intérêt de cet exemple réside peut-être dans le fait que ceux qui se servent du cordonnier ont compris qu'il pouvait être utile d'aller chercher un homme d'origine humble pour séduire un auditoire très éloigné des cercles de pouvoirs tout comme un public d'érudits conquis par le personnage haut en couleur. Les prophètes les plus souvent cités par Salimbene lorsqu'il nous parle de l'Asdente sont la Sybille et les inséparables Merlin et Joachim de Flore. Notre auteur franciscain insiste sur le fait que les gens venaient des quatre coins du monde pour écouter le cordonnier interpréter l'avenir.

Jean-Patrice Boudet rappelle que lorsque Dante décrira ce prophète dans la quatrième bolge du huitième cercle de L'Enfer, là où se trouvent les devins et les sorciers, il mentionnera aussi toutes ces femmes qui, pour leur malheur, se firent devineresses en délaissant aiguille, navette et fuseau ${ }^{37}$. Les prophéties n'intéressent donc pas que les hommes d'Église, même s'ils restent les plus fervents collectionneurs de compilations de prédictions. Elles ne séduisent pas qu'à la cour des puissants, elles savent aussi intriguer les plus faibles, jusqu'à les pousser à se faire eux-mêmes prophètes. Ainsi les puissants ont tout intérêt à trouver des versions orales des prophéties savantes capables de plaire à tous ceux qui n'ont que pas ou peu d'instruction. La prophétie merlinienne, langage politique complexe, trouve un écho auprès du peuple, en Grande-Bretagne, en France et en Italie, et participe à la construction de références culturelles politisées.

Les prophéties de Merlin nous permettent donc d'appréhender une forme de communication politique entre les élites et le peuple. Le langage politique savant, qu'il soit originellement conçu par des dissidents comme des franciscains joachimites, ou bien par le pouvoir en place, sous l'action des propagandistes d'État, est alors dégradé, simplifié, pour être compris par

37. J.-P. BoudeT, Entre science et nigromance. Astrologie, divination et magie dans l'Occident médiéval (XII ${ }^{e}-X V^{e}$ s.), Paris, 2006, p. 13. 
le peuple. Convaincre les milieux populaires s'avère important à la fin du Moyen Âge, au moment de la naissance des nations, pour assurer une cohésion sociale autour du souverain et du royaume. À la même époque se développent les mythes des origines des peuples, censés apporter des spécificités glorieuses à chaque peuple en lui assurant une indépendance incontestable ${ }^{38}$.

Mais faut-il en déduire que la communication politique se réduit alors à une communication descendante, du plus fort vers le plus faible ou du plus savant vers l'illettré, qui se conformerait au message envoyé en l'interprétant comme on le lui demande ? Il semblerait que l'appropriation populaire des prédictions ne soit pas exempte de «braconnage culturel» pour reprendre l'expression de Michel de Certeau ${ }^{39}$. Ainsi, la prophétie du Bois Chesnu, transposition populaire d'une prophétie savante, montre des spécificités qui la différencient du texte latin, et qui lui ont assuré une bien plus large audience qu'une simple lecture du texte originel. De même, la prophétie de Lechlavar, la pierre qui parle et qui tue, fait parler d'elle dans les milieux de cour alors qu'elle n'est rien d'autre qu'une prophétie populaire qui ne s'appuie sur aucun texte latin connu avant que Giraud ne la transcrive. La communication n'est donc pas seulement descendante. Rumeurs populaires et textes savants sont plutôt interdépendants.

Enfin, s'il est facile de comptabiliser les manuscrits comportant des prophéties pour essayer d'en déduire leur audience, il est problématique de recenser les voies de diffusion des versions orales des prophéties, propagées certes par la prédication, par les ménestrels itinérants, mais aussi par d'autres canaux informels que nous avons bien du mal à appréhender. L'efficacité est cependant au rendez-vous, comme nous l'avons vu avec l'exemple des mobilisations panceltes au nom de Merlin qui jalonnent l'histoire de la Grande-Bretagne, ou avec celui de l'Italie du Nord où les prophéties du devin sont présentes sur tous les fronts, et sont tellement imbriquées aux contextes locaux et aux luttes entre les cités qu'elles demeurent souvent incompréhensibles.

La présence de prophéties identiques dans des manuscrits datés du XII ${ }^{\mathrm{e}}$ $\mathrm{au} \mathrm{XV}$ siècle peut suffire à montrer leur influence dans les milieux de cour ou les milieux cléricaux. Pour témoigner de la longévité des rumeurs prophétiques et de l'audience de Merlin en milieu populaire, je citerai un compte rendu datant du $\mathrm{XVI}^{\mathrm{e}}$ siècle et figurant dans le manuscrit Lansdowne 111,

38. Ainsi l'Écosse s'invente des origines égyptiennes pour contrer le mythe des racines troyennes de l'Angleterre et ses revendications sur l'Écosse en vertu du partage de l'île de Bretagne effectué au temps de Brutus. La construction du mythe des origines pharaoniques de l'Écosse débute au XIV ${ }^{\mathrm{e}}$ siècle et se poursuit jusqu'au XVI ${ }^{\mathrm{e}}$ siècle. E. L. G. STONES, Anglo-Scottish relations (1174-1328) : Some selected Documents, Oxford, 1970, p. 226 ; Pierre de LANGTOFT, Le règne d'Édouard I ${ }^{e r}$, éd. J.-C. Thiolier, Créteil, 1989, p. 450 ; W. MATthews « The Egyptians in Scotland: The political History of a Myth », Viator, I, 1970, p. 289-306; A. MASON «Scotching the Brut: Politics, History and National Myth in Sixteenth century Britain », dans Scotland and England, 1286-1815, Edimbourg, 1985, p. 60-84.

39. M. De Certeau, L. Giard, P. Mayol, L'Invention du quotidien, t. 1 et 2, Paris, 1990. 
compilation de documents appartenant à William Cecil, lord Burghley, conseiller d'Élisabeth $\mathrm{I}^{\mathrm{re}}$, et concernant le pays de Galles, l'Écosse, l'Irlande et les îles anglo-normandes. Ce rapport traite des ignorances en matière de religion des Gallois du Nord et montre que le peuple gallois se considérait encore, à la fin $\mathrm{du} \mathrm{XVI}$ siècle, longtemps après la fin de l'indépendance galloise, comme une nation en captivité, une nation qui se souvient des vaticinations de Merlin et des guerres contre l'Angleterre qui structurent encore son identité :

Upon the Sundays and Holidays the multitude of all sorts of men, women, and children of every parish do use to meet in sundry places, either on some hill or on the side of some mountain where their Harpers and Crowthers sing them songs of the doings of their Ancestors; namely of their wars against the Kings of this realme, and the English nation : and then do they rip up their pedigrees at length, how each of them is descended from their old Princes. Here also do they spend their time in hearing some part of the lives of Thalaassyn, Marlin, Beno, Rybbye, Jermin, and such other the intended Prophets and Saints of that country ${ }^{40}$.

Le lien entre l'ancienne aristocratie dirigeante devenue mythique et le peuple n'est pas rompu, et les rêves d'indépendance s'articulent toujours autour des noms des saints et des prophètes, dont Merlin et son homologue Taliesin, qui assurent encore une forme de lien social jugé subversif par le pouvoir anglais. L'audience de Merlin est encore forte en milieu populaire à la fin $\mathrm{du} \mathrm{XVI}^{\mathrm{e}}$ siècle pour des raisons politiques, à l'époque où l'historicité de la légende arthurienne est pourtant sérieusement mise à mal.

Catherine DANIEL, Université Rennes 2, UFR ALC, Département des Lettres Place du recteur Henri le Moal, CS 24307 - 35043 Rennes cedex

\section{L'audience des prophéties de Merlin : entre rumeurs populaires et textes savants}

L'article étudie la réception des prophéties de Merlin en dehors des cercles de pouvoir et d'érudition pour comprendre comment une audience populaire pouvait appréhender des prédictions de nature complexe, volontairement obscures. La communication politique entre puissants et anonymes est assurée par la circulation de rumeurs prophétiques appuyées sur des versions écrites savantes qui fondent leur autorité.

Merlin - prophéties - propagande - rumeurs

40. The state of North Wales touching religion, 1575, Londres, British, Library, ms. Lansdowne 111, art. 4. Original letters illustrative of English History, H. ElLIS éd., Londres, 1846, p. 49. 
The Audience of the Prophecies of Merlin : from Popular Rumour to Scholarly Texts

This article looks at the way Merlin's prophecies were perceived by those other than the powerful and learned, so as to understand how ordinary people could comprehend these complex, deliberately obscure, predictions. Political communication between the powerful and the anonymous was ensured by the circulation of prophetic rumours deriving from learned written texts which gave them credibility.

Merlin - prophecies - propaganda - rumours 
